\title{
Trajectory Dynamics Study of the $\mathrm{Ar}+\mathrm{CH}_{4}$ Dissociation Reaction at High Temperatures: the Importance of Zero-Point-Energy Effects
}

\author{
J. M. C. Marques, ${ }^{*}, \dagger$ E. Martínez-Núñez, ${ }^{\ddagger}$ A. Fernández-Ramos, ${ }^{\ddagger}$ and S. A. Vázquez \\ Departamento de Química, Universidade de Coimbra, 3004-535 Coimbra, Portugal, and Departamento de \\ Química Física, Universidad de Santiago de Compostela, Santiago de Compostela E-15782, Spain
}

Received: November 19, 2004; In Final Form: January 5, 2005

\begin{abstract}
Large-scale classical trajectory calculations have been performed to study the reaction $\mathrm{Ar}+\mathrm{CH}_{4} \rightarrow \mathrm{CH}_{3}+$ $\mathrm{H}+\mathrm{Ar}$ in the temperature range $2500 \leq T / \mathrm{K} \leq 4500$. The potential energy surface used for $\mathrm{ArCH}_{4}$ is the sum of the nonbonding pairwise potentials of Hase and collaborators $(J$. Chem. Phys. 2001, 114, 535) that models the intermolecular interaction and the $\mathrm{CH}_{4}$ intramolecular potential of Duchovic et al. (J. Phys. Chem. 1984, 88, 1339), which has been modified to account for the $\mathrm{H}-\mathrm{H}$ repulsion at small bending angles. The thermal rate coefficient has been calculated, and the zero-point energy (ZPE) of the $\mathrm{CH}_{3}$ product molecule has been taken into account in the analysis of the results; also, two approaches have been applied for discarding predissociative trajectories. In both cases, good agreement is observed between the experimental and trajectory results after imposing the $\mathrm{ZPE}$ of $\mathrm{CH}_{3}$. The energy-transfer parameters have also been obtained from trajectory calculations and compared with available values estimated from experiment using the master equation formalism; in general, the agreement is good.
\end{abstract}

\section{Introduction}

Collision-induced dissociation (CID) processes have long been subject to many studies in various contexts. The CID mechanism may occur through direct dissociation (e.g., as the result of a high energetic collision) where the molecule is fragmented during the initial impact or, in two steps, with the prior formation of an internally excited species followed by its unimolecular dissociation. In both cases, the amount of collisional energy transfer is a key parameter in the study of such reactions. Indeed, knowledge of the mean collisional energy transfer is imperative in using the Rice-Ramsperger-KasselMarcus (RRKM) theory ${ }^{1,2}$ to calculate rate constants for dissociation (or recombination) reactions. This becomes especially important in combustion chemistry, where the dissociation (or even recombination) reactions occur at high temperatures and low pressures. The RRKM theory uses estimates of the mean collisional energy transfer (which are normally obtained from experimental results on related systems at a particular temperature) to calculate the rate constants for such reactions. Because low-pressure rate constants are quite sensitive to collisional energy transfer, those RRKM calculations may occasionally lead to inaccurate values.

The dissociation reaction

$$
\mathrm{M}+\mathrm{CH}_{4} \rightarrow \mathrm{CH}_{3}+\mathrm{H}+\mathrm{M}
$$

where $\mathrm{M}$ is usually a rare gas atom (e.g., argon), plays a fundamental role in combustion. Under most experimental concentrations of $\mathrm{CH}_{4}$, reaction 1 can compete with reaction $\mathrm{H}$ $+\mathrm{CH}_{4} \rightarrow \mathrm{CH}_{3}+\mathrm{H}_{2}$, which is clearly important for the $\mathrm{CH}_{4} /$ $\mathrm{O}_{2}$ combustion chemistry. ${ }^{3}$ Because of this, reaction 1 has been

* Author to whom correspondence should be sent. E-mail: qtmarque@ ci.uc.pt.

$\doteqdot$ Universidade de Coimbra.

$\doteqdot$ Universidad de Santiago de Compostela. subjected to many experimental ${ }^{4-9}$ and theoretical ${ }^{10,11}$ studies. Most of these studies focused their attention on the determination (or calculation) of the dissociation rate coefficients in the hightemperature regime. In particular, Kiefer and Kumaran ${ }^{7}$ have performed laser-schlieren shock-tube experiments in the temperature range $2800 \leq T / \mathrm{K} \leq 4300$ to measure the rate of dissociation of $\mathrm{CH}_{4}$ diluted in both $\mathrm{Ar}$ and $\mathrm{Kr}$. They have concluded that the thermal rate coefficient of reaction 1 should show significant Arrhenius curvature at high temperatures. ${ }^{7}$ More recently, Sutherland et al. ${ }^{12}$ have used atomic-resonance absorption spectrometry to observe absolute $\mathrm{H}$-atom formation rates from $\mathrm{CH}_{4}$ dissociation with $\mathrm{Kr}$ as a bath gas. These experimental results ${ }^{12}$ confirmed those obtained by Kiefer and Kumaran, ${ }^{7}$ while establishing that the collisional-energy-transfer parameter in $\mathrm{Kr}$ increases with increasing temperature; they ${ }^{12}$ expect relatively little difference in the energy-transfer parameter when $\mathrm{Kr}$ is replaced by $\mathrm{Ar}$ as the bath gas. Moreover, from the theoretical side, Miller et al. ${ }^{11}$ have used three formulations of the master equation, applied to methane dissociation in the lowpressure limit, to extract information about collisional energy transfer. Thus, the values of the collisional energy transfer deduced $^{11}$ from experimental rate coefficients ${ }^{7}$ are shown to increase continuously as the temperature increases from 300 to $4000 \mathrm{~K}$

The quasi-classical trajectory (QCT) method has proven to be reliable for studying energy transfers in highly excited molecules, ${ }^{13,14}$ and, in particular, it has been applied to investigate the energy transfer in collisions of argon with methane. ${ }^{15-17}$ However, in comparison with the present work, all of these studies ${ }^{15-17}$ have used less realistic analytical models to represent both the intramolecular $\mathrm{CH}_{4}$ and the intermolecular $\mathrm{Ar}-\mathrm{CH}_{4}$ interactions. Besides, the QCT method has the clear advantage of having no approximation in the dynamics inherent to the energy-transfer process (other than the use of classical mechanics) while allowing the calculation of the relevant parameters without using any kind of experimental data as input. 
In turn, it also allows the calculation of thermal rate coefficients for reaction 1. Of course, the trajectory approach suffers from some drawbacks, namely, those related to quantum-dynamics effects, and its application to systems with many degrees of freedom is limited by the knowledge of the corresponding potential energy surface and the computational means available. Concerning the quantum effects, one expects tunneling to have a minor influence on the title reaction, once it is barrierless along the minimum energy path. Conversely, as a result of the high endoergicity of reaction 1, the problem of zero-point energy (ZPE) conservation in the $\mathrm{CH}_{3}$ product should be the most relevant disadvantage of using classical trajectories, even in the combustion range of temperatures. However, many strategies have been proposed ${ }^{18-35}$ to approximately correct for this problem. Among these, some are designated as active, because they seek to correct the ZPE leakage along the integration of each trajectory, while the others take into account only the energies of reactant and product molecules (and sometimes that of the transition-state structure) and, hence, are called passive methods. Because the latter do not interfere in the dynamics of the trajectories (they only "manipulate" the statistics of the ensemble of trajectories according to some predefined criteria), they are more convenient from both a practical implementation and theoretical justification; see refs 34 and 36-41 for a critical analysis of the ZPE problem in classical trajectory calculations.

Our main goal is to perform a classical trajectory study of the title reaction in the combustion range of temperatures while assessing the ZPE effects in the thermal rate coefficients; after ZPE correction, the calculated thermal rate coefficients can be compared with the experimental ones. To our knowledge, this is the first theoretical dynamics study attempting to calculate the thermal rate constant for reaction 1 . We also want to estimate the $\mathrm{Ar}+\mathrm{CH}_{4}$ collisional energy-transfer parameters at those high temperatures. Thus, the plan of this paper is as follows. In section 2, we give the details about the $\mathrm{Ar}-\mathrm{CH}_{4}$ potential energy surface and describe the process of choosing initial conditions for trajectory calculations. The trajectory analysis (including the way in which the $\mathrm{ZPE}$ of $\mathrm{CH}_{3}$ is imposed) and the results for both thermal rate constants and energy-transfer parameters are presented and discussed in section 3. Finally, section 4 gathers the main conclusions.

\section{Trajectory Calculations}

2.1. Potential Energy Surface. The potential energy surface for $\mathrm{ArCH}_{4}$ may be written as

$$
V_{\mathrm{ArCH}_{4}}=V_{\mathrm{CH}_{4}}+\sum_{i=1}^{5} V_{\mathrm{Ar}-\mathrm{X}^{i}}
$$

where $V_{\mathrm{CH}_{4}}$ is the full-dimensional potential function for methane and the five $V_{\mathrm{Ar}-\mathrm{X}^{i}}\left(\mathrm{X}^{1}\right.$ represents the carbon atom, while $\mathrm{X}^{2}$, $\mathrm{X}^{3}, \mathrm{X},{ }^{4}$ and $\mathrm{X}^{5}$ stand for the hydrogens) pair potentials account for the $\mathrm{Ar}-\mathrm{CH}_{4}$ van der Waals interaction. We have employed the recent intermolecular $\mathrm{Ar}-\mathrm{CH}_{4}$ potential of Hase and collaborators, ${ }^{42}$ which is expected to perform well for the range of temperatures studied in this work. This has been calibrated with QCISD(T)/6-311++ $G^{* *}$ data, ${ }^{42}$ and the corresponding two-body functions have the form

$$
V_{\mathrm{Ar}-\mathrm{X}}(R)=A \exp (-b R)+C / R^{6}
$$

where the $A, b$, and $C$ parameters for both $\mathrm{Ar}-\mathrm{C}$ and $\mathrm{Ar}-\mathrm{H}$ interactions have been taken from the potential identified as $\mathrm{Ar} / \mathrm{CH}_{4}-\mathrm{III}$ in ref 42 ; for other $\mathrm{Ar}-\mathrm{C}$ and $\mathrm{Ar}-\mathrm{H}$ potentials, see refs 43 and 44 . The use of eq 3 must be done with some caution for very short $\mathrm{Ar}-\mathrm{X}$ distances (high energies) where the second term ( $C$ is negative) dominates over the first one, which leads to a negative potential. We have verified that those short distances are far from being reached in this trajectory calculation. In addition, the $\mathrm{Ar} / \mathrm{CH}_{4}-\mathrm{III}$ potential has been shown to provide agreement with high-energy experiments for the $\mathrm{Ar}+\mathrm{C}_{2} \mathrm{H}_{6}$ system. ${ }^{45}$

The potential energy surface adopted in this work for $\mathrm{CH}_{4}$ is the one of Duchovic, Hase, and Schlegel ${ }^{46}$ (DHS), which is known to adequately describe the breakage of the $\mathrm{C}-\mathrm{H}$ bond, that is, the dissociation reaction $\mathrm{CH}_{4} \rightarrow \mathrm{CH}_{3}+\mathrm{H}$; this is a key step in the present CID study. However, we have found that the DHS surface allows the $\mathrm{H}$ atoms to closely approach each other when $\mathrm{CH}_{4}$ is highly vibrationally excited, which is not infrequent for the temperatures considered here. Indeed, Hase and collaborators ${ }^{46}$ have already pointed out that their $\mathrm{CH}_{4}$ potential may not be reliable to be used in classical trajectory calculations of methane dissociation at energies where other channels are open [e.g., the reactions $\mathrm{CH}_{4} \rightarrow \mathrm{CH}_{2}\left({ }^{1} \mathrm{~A}_{1}\right)+\mathrm{H}_{2}$ and $\mathrm{CH}_{4} \rightarrow \mathrm{CH}_{2}\left({ }^{3} \mathrm{~B}_{1}\right)+\mathrm{H}_{2}$, having thresholds (cf. ref 46) respectively of $\sim 15$ and $\sim 7 \mathrm{kcal} \mathrm{mol}^{-1}$ higher than that for $\left.\mathrm{CH}_{4} \rightarrow \mathrm{CH}_{3}+\mathrm{H}\right]$. Thus, we have accounted for the $\mathrm{H}-\mathrm{H}$ repulsion by adding to the original $\mathrm{CH}_{4}$ potential six terms of the type

$$
V_{\mathrm{H}-\mathrm{H}}(R)=A \exp (-b R)
$$

multiplied by the corresponding switching function

$$
f_{i}\left(R_{\mathrm{H}-\mathrm{H}}\right)=\frac{1}{2}\left\{1-\tanh \left[\gamma\left(R_{\mathrm{H}-\mathrm{H}}-R_{\mathrm{cut}}\right)\right]\right\} \quad(i=1-6)
$$

At small $\mathrm{H}-\mathrm{H}$ distances (for example, $R_{\mathrm{H}-\mathrm{H}}<R_{\text {cut }}$ ), the repulsive potential of eq 4 dominates over all of the other $\mathrm{CH}_{4}$ interactions and disappears for $R_{\mathrm{H}-\mathrm{H}}>R_{\text {cut }}$. We have chosen $R_{\text {cut }}=1.0 \AA$ because this already corresponds to a strong repulsive interaction region of the DHS surface. Moreover, a rapid switch from the pure repulsive potential given by eq 4 and the DHS function is necessary to reproduce the original $\mathrm{CH}_{4}$ frequencies at its equilibrium geometry; $\gamma=100 \AA^{-1}$ has been found to be a good choice for the decaying parameter of the switching function in eq 5 . This new potential function that accounts for the drawback of the DHS surface was then fitted to $\mathrm{QCISD}(\mathrm{T}) / 6-311++\mathrm{G}(\mathrm{d}, \mathrm{p})$ ab initio points calculated for 10 different $\mathrm{HCH}$ angles, and the resulting parameters in eq 4 are $A=1400 \mathrm{kcal} \mathrm{mol}^{-1}$ and $b=8.5 \AA^{-1}$. Figure 1 shows both the DHS surface ${ }^{46}$ and the improved $\mathrm{CH}_{4}$ potential as functions of the $\mathrm{HCH}$ angle. It is clear from this figure that the improved $\mathrm{CH}_{4}$ surface reproduces quite well the present ab initio calculations, while the DHS one is in complete disagreement for the smallest angles.

Figure 2 shows a contour plot for Ar moving around the $\mathrm{CH}_{4}$ molecule, which has been fixed at its equilibrium geometry. The topographical details of the intermolecular potential energy surface that stand out in this figure result just from a cut of the global potential energy surface, which corresponds to argon moving in the plane formed by carbon and two of the hydrogen atoms. Because of this, only two of the four equivalent minima $\left(E_{\min }=-0.20 \mathrm{kcal} \mathrm{mol}^{-1}\right.$ below the $\mathrm{Ar}+\mathrm{CH}_{4}$ asymptotic limit $)$ are visible in Figure 2 (shaded areas). These are associated with lines of the $\mathrm{Ar}-\mathrm{C}$ approach that are perpendicular to the four faces of the tetrahedron, with an argon-carbon distance of $R_{\mathrm{Ar}-\mathrm{C}}$ $=4.12 \AA$. Also shown by the dots in the figure are two of the saddle points for isomerization $\left(E_{\text {saddle }}=-0.13 \mathrm{kcal} \mathrm{mol}^{-1}\right)$ 


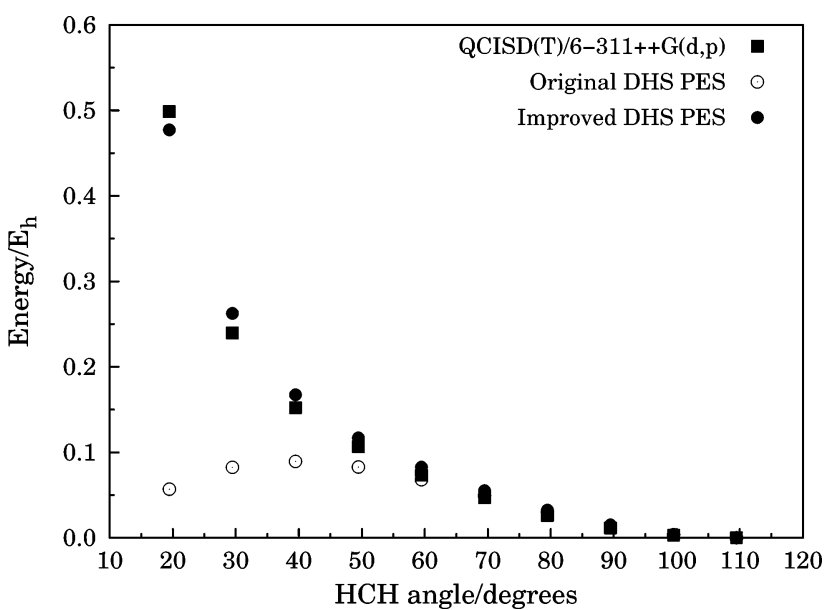

Figure 1. Comparisons of the improved DHS potential energy surface (full circles) and the original DHS potential energy surface (open circles) with the QCISD(T)/6-311++G(d,p) ab initio calculations (full squares) as a function of the $\mathrm{HCH}$ angle.

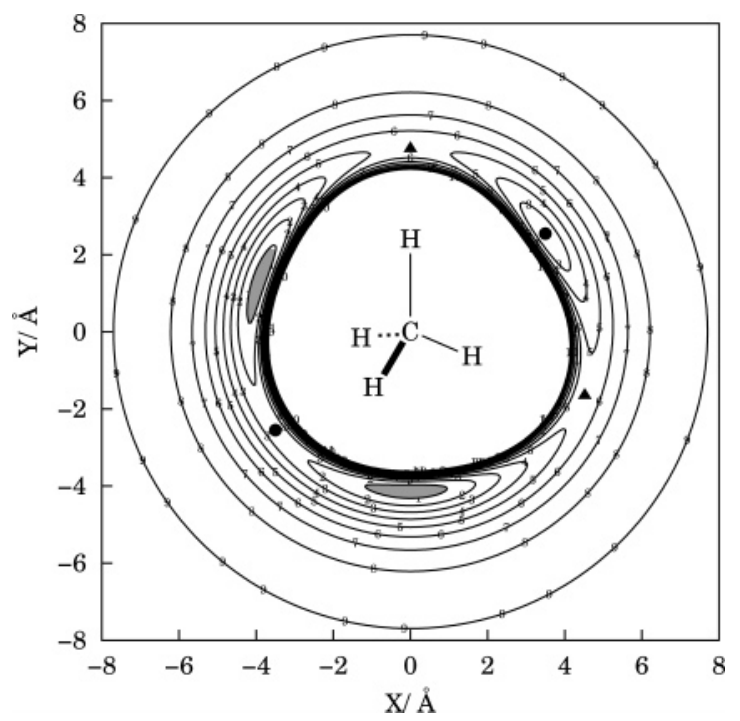

Figure 2. Contour energy plot for the $\mathrm{Ar}$ atom around the $\mathrm{CH}_{4}$ molecule (fixed in its equilibrium geometry) in the plane defined by the $\mathrm{C}-\mathrm{H}$ bonds that are indicated with the thin lines. The $\mathrm{C}-\mathrm{H}$ bonds standing up and down that plane are represented by the thick and dotted lines, respectively. The first energy contour corresponds to $E_{1}=-0.22$ $\mathrm{kcal} \mathrm{mol}^{-1}, \Delta E=0.02 \mathrm{kcal} \mathrm{mol}^{-1}$ being the energy difference between contours. The shaded areas show the minima, while full dots and triangles stand for first-order and second-order saddle points, respectively. See the text for further explanation.

that correspond to the edges of the tetrahedron $\left(R_{\mathrm{Ar}-\mathrm{C}}=4.30\right.$ $\AA)$; the second-order saddle points $\left(E_{\text {saddle }, 2}=-0.07 \mathrm{kcal} \mathrm{mol}^{-1}\right)$ at the vertices $\left(R_{\mathrm{Ar}-\mathrm{C}}=4.78 \AA\right)$ are indicated by triangles in Figure 2. Note that, although deeper (i.e., $E_{\min }=-0.39 \mathrm{kcal}$ $\mathrm{mol}^{-1}, E_{\text {saddle }}=-0.32 \mathrm{kcal} \mathrm{mol}^{-1}$, and $E_{\text {saddle }, 2}=-0.24 \mathrm{kcal}$ $\left.\mathrm{mol}^{-1}\right)$, similar $\mathrm{Ar}-\mathrm{CH}_{4}$ structures $\left(R_{\mathrm{Ar}-\mathrm{C}}=3.70 \AA, R_{\mathrm{Ar}-\mathrm{C}}=\right.$ $3.90 \AA$, and $R_{\mathrm{Ar}-\mathrm{C}}=4.25 \AA$, respectively) have been recently obtained in the $\operatorname{CCSD}(\mathrm{T}) /$ aug-cc-pVTZ ab initio calculations of Liu and Jäger ${ }^{47}$ these ab initio values are in very good agreement with those from the symmetry-adapted perturbation theory calculations of Heijmen et al. ${ }^{48}$

We present in Figure 3 the main energetic features of the title reaction in the present $\mathrm{ArCH}_{4}$ potential energy surface. It is clear from this figure that the reaction $\mathrm{Ar}+\mathrm{CH}_{4} \rightarrow \mathrm{CH}_{3}+$ $\mathrm{H}+\mathrm{Ar}$ is highly endoenergetic, with the classical energy threshold being $E_{\mathrm{th}}=110.6 \mathrm{kcal} \mathrm{mol}^{-1}$. The value of 112.1 $\mathrm{kcal} \mathrm{mol}^{-1}$ shown in parentheses is estimated from the
$\mathrm{ArCH}_{4}$ potential energy surface

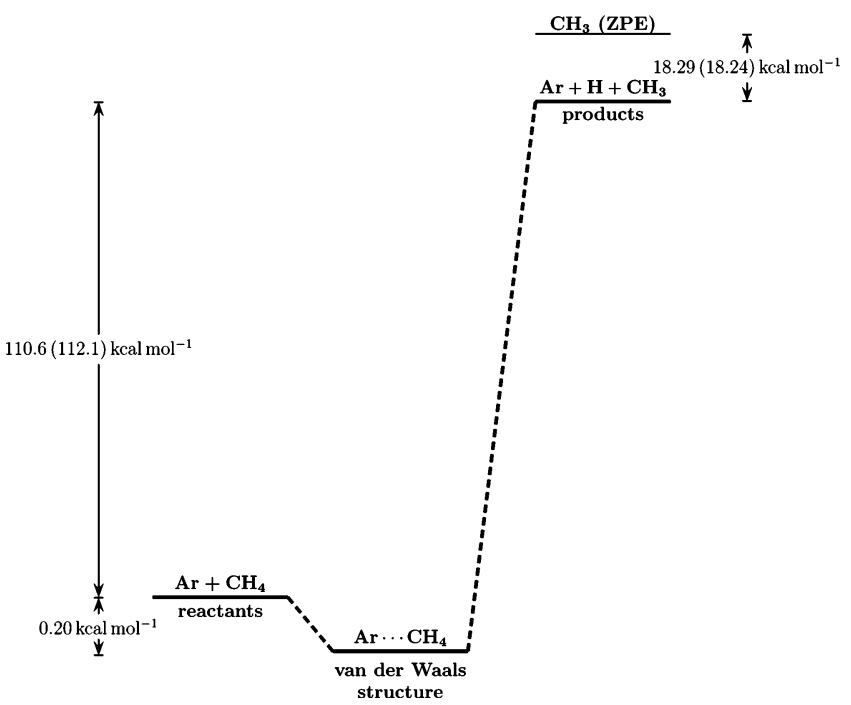

Figure 3. Main energetic features of the reaction $\mathrm{Ar}+\mathrm{CH}_{4} \rightarrow \mathrm{CH}_{3}$ $+\mathrm{H}+$ Ar. Values in parentheses are the experimental estimates. See the text for further explanation.

experimental enthalpies at $298.15 \mathrm{~K}$ obtained at NIST $^{49}$ and taking into account the corresponding thermal energy as well as the ZPEs of both $\mathrm{CH}_{4}$ and $\mathrm{CH}_{3}$. Also indicated in Figure 3 is the $\mathrm{Ar}-\mathrm{CH}_{4}$ van der Waals structure associated with the aforementioned four equivalent minima. Finally, we have shown in this figure the $\mathrm{ZPE}$ of $\mathrm{CH}_{3}$ (i.e., $E_{\mathrm{ZPE}}^{\mathrm{CH}_{3}}=18.29 \mathrm{kcal} \mathrm{mol}^{-1}$ ), which has been calculated using the harmonic frequencies of $\mathrm{CH}_{3}$ arising in a normal-mode analysis of the DHS potential, ${ }^{46}$ while the corresponding experimental value is indicated in parentheses.

2.2. Initial Conditions. The thermal rate coefficients were evaluated by the expression ${ }^{50}$

$$
k(T)=\left(\frac{8 k_{\mathrm{B}} T}{\pi \mu}\right)^{1 / 2} \sigma(T)
$$

where $k_{\mathrm{B}}$ is the Boltzmann constant, $\mu$ is the $\mathrm{Ar}-\mathrm{CH}_{4}$ reduced mass, and $\sigma(T)$ is the reactive cross section at temperature $T$; as usual, $\sigma(T)=\pi b_{\max }{ }^{2} N_{\mathrm{r}} / N$, with $b_{\max }, N_{\mathrm{r}}$, and $N$ being the largest impact parameter that leads to reaction, the number of reactive trajectories, and the total number of trajectories for a given temperature, respectively. The reactive cross sections have been calculated by running trajectories with an extensively adapted version ${ }^{44,51,52}$ of the MERCURY program, ${ }^{53}$ which allows for the definition of all possible reactive channels. ${ }^{44}$ This has been further modified to incorporate the proper selection of initial conditions for the present work that we describe as follows.

The collision energy $E_{\text {tr }}$ has been selected from the MaxwellBoltzmann distribution at temperature $T$ using the appropriate cumulative function ${ }^{18}$

$$
G\left(E_{\mathrm{tr}}\right)=\left(k_{\mathrm{B}} T\right)^{-2} \int_{0}^{E_{\mathrm{tr}}} E_{\mathrm{tr}}^{\prime} \exp \left(-E_{\mathrm{tr}}^{\prime} / k_{\mathrm{B}} T\right) \mathrm{d} E_{\mathrm{tr}}^{\prime}
$$

where the value of $E_{\mathrm{tr}}$ for each trajectory is randomly calculated by numerically solving the equation $G\left(E_{\mathrm{tr}}\right)-\xi=0 ; \xi$ is a random number chosen in the range $0 \leq \xi \leq 1$. In turn, the vibrational normal modes of $\mathrm{CH}_{4}$ have been sampled quasiclassically from the corresponding Boltzmann distributions, as described in detail in ref 50 . 
TABLE 1: Trajectory Calculations for the Study of Reaction $\mathrm{Ar}+\mathrm{CH}_{4} \rightarrow \mathrm{CH}_{3}+\mathrm{H}+\mathrm{Ar}^{a}$

\begin{tabular}{cccccc}
\hline$T / \mathrm{K}$ & $N_{\mathrm{g}}{ }^{b}$ & $f_{\mathrm{ni}}{ }^{c}$ & $T / \mathrm{K}$ & $N_{\mathrm{g}}{ }^{b}$ & $f_{\mathrm{ni}}{ }^{c}$ \\
\hline 2500 & $6 \times 10^{6}$ & 0.987 & 3800 & $1 \times 10^{5}$ & 0.740 \\
2800 & $1 \times 10^{6}$ & 0.963 & 4000 & $4 \times 10^{4}$ & 0.677 \\
3000 & $5 \times 10^{5}$ & 0.936 & 4200 & $4 \times 10^{4}$ & 0.614 \\
3200 & $5 \times 10^{5}$ & 0.899 & 4500 & $4 \times 10^{4}$ & 0.524 \\
3500 & $2.5 \times 10^{5}$ & 0.827 & & &
\end{tabular}

${ }^{a}$ See the text. ${ }^{b}$ Number of generated trajectories. ${ }^{c}$ Fraction of the generated trajectories that have been nonintegrated.

The sampling of rotational energy and angular momentum for $\mathrm{CH}_{4}$ takes into account that the molecule is a spherical top with equal momenta of inertia according to the three principal axes of inertia (i.e., $I \equiv I_{\mathrm{a}}=I_{\mathrm{b}}=I_{\mathrm{c}}$ ). The rotational energy for a spherical top depends only on the rotational angular momentum $J$ (i.e., it has no contribution from the angular momentum component along the molecule-fixed axis) so that $J$ is sampled from the probability distribution

$$
P(J)=\frac{J}{I k_{\mathrm{B}} T} \exp \left(-J^{2} / 2 I k_{\mathrm{B}} T\right)
$$

where $0 \leq J \leq \infty$ and $P(J)$ is already normalized. Thus, $J$ may be sampled using the cumulative distribution function, which, after integration, leads to the result

$$
J=\left[-2 I k_{\mathrm{B}} T \ln \left(1-\xi_{1}\right)\right]^{1 / 2}
$$

Moreover, all three of the components of $J$ are treated on equal footing; that is

$$
\begin{gathered}
J_{x}=J \cos \left(2 \pi \xi_{2}\right) \sin \left(\pi \xi_{3}\right) \\
J_{y}=J \sin \left(2 \pi \xi_{2}\right) \sin \left(\pi \xi_{3}\right) \\
J_{z}=J \cos \left(\pi \xi_{3}\right)
\end{gathered}
$$

and the classical rotational energy is calculated as $E_{\mathrm{rot}}=J^{2} / 2 I$; the random numbers $\xi_{i}(i=1-3)$ belong to the range $0 \leq \xi_{i}$ $\leq 1$.

Once the initial conditions are established, one of two situations may arise: (i) the total energy $\left(E_{\mathrm{tot}}=E_{\mathrm{vib}}+E_{\mathrm{rot}}+\right.$ $\left.E_{\mathrm{tr}}\right)$ is below the dissociation threshold (i.e., $E_{\mathrm{tot}}<E_{\mathrm{th}}$ ), or (ii) $E_{\mathrm{tot}} \geq E_{\mathrm{th}}$. In case $\mathrm{i}$, the trajectory has no chance of forming products and, hence, it is not integrated, being considered nonreactive. Because of the endoergicity of the reaction, this is the most frequent case, as shown by the fraction of such trajectories in column 3 of Table 1. Thus, from the total number of generated trajectories $\left(N_{\mathrm{g}}\right)$ presented in Table 1 , only a very small subset (corresponding to those with initial conditions described in case ii) needs to be integrated until one or more internuclear distances reach $10 \AA$ or 90 ps has elapsed. In all cases, the maximum impact parameter $\left(b_{\max }\right)$ has been fixed at $4 \AA$. This is well-optimized for the main calculations of the present work, where the ZPE effects have been taken into account (see section 3.1).

Concerning the energy transfer, we have calculated batches of 20000 trajectories for temperatures $T=2500,3000,3500$, 4000 , and $4500 \mathrm{~K}$. For each batch, the collision energy has been sampled from the corresponding Boltzmann distribution (see above) while the internal energy of $\mathrm{CH}_{4}$ has been fixed at the threshold (i.e., $110.6 \mathrm{kcal} \mathrm{mol}^{-1}$ ). The rotational counterpart of the latter has been calculated as $R T / 2$ ( $R$ is the gas constant) for each axis of inertia and the remaining vibrational energy attributed to the normal modes according to a microcanonical distribution ${ }^{54}$ thus, the initial vibrational (rotational) energies are, in kcal mol${ }^{-1}, 103.2$ (7.4), 101.6 (9.0), 100.2 (10.4), 98.7 (11.9), and 97.2 (13.4) for $T=2500,3000,3500,4000$, and $4500 \mathrm{~K}$, respectively. The end of trajectories is detected when all of the distances between argon and the other atoms become larger than $15 \AA$, which is sufficient to ensure that the energytransfer process is concluded. In turn, $b_{\max }$ has been optimized by using the procedure of Lim and Gilbert, ${ }^{55}$ assuming as criterion of convergence that the second moment of the energy transfer becomes less than $2 \times 10^{-2} \mathrm{~cm}^{-2}$ for $b>b_{\max }$. Because $b_{\text {max }}$ thus calculated should include all of the inelastic collisions, the values obtained are always greater than the corresponding ones for reactive events (i.e., $b_{\max }=4 \AA$ ): $b_{\max }=5.8,5.6$, 5.4, 5.3, and $5.4 \AA$ for $T=2500,3000,3500,4000$, and 4500 $\mathrm{K}$, respectively. Note that $b_{\max }$ is extremely dependent on the potential energy surface; for example, Hase et al. ${ }^{16}$ have used $b_{\text {max }}=4.5$ and $5 \AA$ to study the $\mathrm{Ar}+\mathrm{CH}_{4}$ collisional energy transfer with different $\mathrm{ArCH}_{4}$ model potentials.

\section{Results and Discussion}

3.1. Trajectory Analysis. The contribution to the thermal rate constant of the title reaction may arise from (i) the direct formation of $\mathrm{Ar}+\mathrm{H}+\mathrm{CH}_{3}$ (which is a rare event for endoenergetic processes and has not been observed in the present trajectory calculation), (ii) the formation of a vibrationalrotational excited $\mathrm{CH}_{4}^{*}$ species (due to collision with $\mathrm{Ar}$ ) that can dissociate afterward, and (iii) $\mathrm{CH}_{4}^{*}$ reactants initially prepared with an internal energy above the dissociation threshold. Sometimes the latter should be considered predissociative because they may dissociate prior to the collision with argon. However, a rigorous predissociation criterion cannot be easily established and, hence, it may be assumed that all trajectories initiated with internal energies above the threshold should be discarded from the final statistical analysis; for example, a similar procedure has been adopted by Rodrigues and Varandas $^{43}$ in the $\mathrm{Ar}+\mathrm{HCN}$ collision-induced dissociation. Of course, this criterion for defining predissociative trajectories (hereafter referred to as PD1) should be regarded as an approximation because highly energized $\mathrm{CH}_{4}^{*}$ species may last for a sufficiently long period so that a collision with argon is able to occur. Besides the PD1 procedure, we have applied the RRKM theory ${ }^{1,2}$ to calculate the probability of a trajectory to be predissociative; that is

$$
P\left(t_{\mathrm{col}}\right)=1-\exp \left[-k_{\mathrm{RRKM}}(E) \times t_{\mathrm{col}}\right]
$$

where $k_{\mathrm{RRKM}}(E)$ is the variational RRKM rate constant and $t_{\mathrm{col}}$ $(=600 \mathrm{ps})$ is the average collision time calculated by the kinetic theory of gases for the experimental conditions of pressure and temperature adopted by Kiefer and Kumaran; ${ }^{7}$ this method of assigning predissociative trajectories will be hereafter referred to as PD2. Although this must still be seen as an approximation, it is clearly a better estimation for predissociative trajectories.

Because the title reaction is highly endoenergetic and the nascent $\mathrm{CH}_{3}$ product molecule has a substantial ZPE content (see Figure 3), we expect that important effects due to ZPE leakage may occur in this system. To investigate this, we have considered three cases in the analysis of the trajectories, those being the results presented in Tables 2-4. First, the pure classical analysis considers that all of the trajectories leading to $\mathrm{CH}_{4}^{*}$ species with $E_{\text {int }} \geq 110.6 \mathrm{kcal} \mathrm{mol}^{-1}$ will promptly dissociate to form $\mathrm{H}+\mathrm{CH}_{3}$; the corresponding results are shown in Table 2. 
TABLE 2: Results for the $\mathrm{Ar}+\mathrm{CH}_{4}$ Reaction Obtained through the Pure Classical Analysis of the Trajectory Calculations and the PD2 Method for Defining Predissociative Trajectories ${ }^{a}$

\begin{tabular}{cllll}
\hline$T / \mathrm{K}$ & \multicolumn{1}{c}{$N_{\text {tot }}$} & \multicolumn{1}{c}{$N_{\mathrm{CH}_{4}^{*}}$} & \multicolumn{1}{c}{$10^{2} \sigma_{\mathrm{CID}} / \AA^{2}$} & \multicolumn{1}{c}{$k(T) / 10^{9} \mathrm{~cm}^{3} \mathrm{~s}^{-1} \mathrm{~mol}^{-1}$} \\
\hline 2500 & $5995964(5985768)$ & $10416(870)$ & $8.73 \pm 0.08(0.73 \pm 0.02)$ & $1130.8 \pm 11.1(94.6 \pm 3.2)$ \\
2800 & $996972(991282)$ & $5770(464)$ & $29.09 \pm 0.38(2.35 \pm 0.11)$ & $3987.1 \pm 52.3(322.5 \pm 15.0)$ \\
3000 & $496682(491432)$ & $5318(385)$ & $53.82 \pm 0.73(3.94 \pm 0.20)$ & $7635.1 \pm 104.1(558.6 \pm 28.5)$ \\
3200 & $493613(484872)$ & $8870(706)$ & $90.32 \pm 0.95(7.32 \pm 0.28)$ & $13234.2 \pm 139.2(1072.4 \pm 40.3)$ \\
3500 & $242764(234907)$ & $7929(593)$ & $164.17 \pm 1.81(12.69 \pm 0.52)$ & $25156.6 \pm 277.9(1944.4 \pm 79.7)$ \\
3800 & $94420(89664)$ & $4862(412)$ & $258.83 \pm 3.62(23.10 \pm 1.14)$ & $41326.4 \pm 577.2(3687.7 \pm 181.3)$ \\
4000 & $36810(34494)$ & $2326(168)$ & $317.62 \pm 6.37(24.48 \pm 1.88)$ & $52030.5 \pm 1044.2(4010.3 \pm 308.6)$ \\
4200 & $35719(32928)$ & $2822(236)$ & $397.12 \pm 7.17(36.03 \pm 2.34)$ & $66660.2 \pm 1204.2(6047.2 \pm 392.2)$ \\
4500 & $33723(30222)$ & $3544(280)$ & $528.25 \pm 8.39(46.57 \pm 2.77)$ & $91782.1 \pm 1458.5(8091.4 \pm 481.3)$
\end{tabular}

${ }^{a}$ Values in parentheses are obtained through the PD1 approach for assigning predissociative trajectories; see the text.

TABLE 3: Results for the Ar + $\mathrm{CH}_{4}$ Reaction Using the "ZPE Analysis" and the PD2 Method for Defining Predissociative Trajectories $^{a}$

\begin{tabular}{cllclll}
\hline$T / \mathrm{K}$ & \multicolumn{1}{c}{$N_{\text {tot }}$} & $N_{\mathrm{CH}_{4}^{*}}$ & $N_{600}$ & $N_{\text {diss }}$ & $10^{3} \sigma_{\mathrm{CID}} / \AA^{2}$ & $k(T) / 10^{9} \mathrm{~cm}^{3} \mathrm{~s}^{-1} \mathrm{~mol}^{-1}$ \\
\hline 2500 & $5998755(5998724)$ & $105(88)$ & $33(31)$ & $17(16)$ & $0.14 \pm 0.03(0.13 \pm 0.03)$ & $1.8 \pm 0.4(1.7 \pm 0.4)$ \\
2800 & $998879(998850)$ & $93(78)$ & $38(37)$ & $18(14)$ & $0.90 \pm 0.21(0.70 \pm 0.19)$ & $12.4 \pm 2.9(9.7 \pm 2.6)$ \\
3000 & $498576(498544)$ & $108(80)$ & $42(39)$ & $15(11)$ & $1.51 \pm 0.39(1.11 \pm 0.33)$ & $21.4 \pm 5.5(15.7 \pm 4.7)$ \\
3200 & $496955(496903)$ & $213(183)$ & $63(59)$ & $36(35)$ & $3.64 \pm 0.61(3.54 \pm 0.60)$ & $53.4 \pm 8.9(51.9 \pm 8.8)$ \\
3500 & $246167(246099)$ & $242(194)$ & $92(88)$ & $43(39)$ & $8.78 \pm 1.34(7.97 \pm 1.28)$ & $134.5 \pm 20.5(122.1 \pm 19.5)$ \\
3800 & $96850(96803)$ & $187(156)$ & $69(65)$ & $31(28)$ & $16.09 \pm 2.89(14.54 \pm 2.75)$ & $256.9 \pm 46.1(232.1 \pm 43.9)$ \\
4000 & $38081(38061)$ & $103(91)$ & $39(38)$ & $20(18)$ & $26.40 \pm 5.90(23.77 \pm 5.60)$ & $432.4 \pm 96.7(389.4 \pm 91.8)$ \\
4200 & $37277(37245)$ & $137(114)$ & $52(52)$ & $38(34)$ & $51.24 \pm 8.31(45.89 \pm 7.87)$ & $860.1 \pm 139.5(770.2 \pm 132.0)$ \\
4500 & $35786(35740)$ & $202(172)$ & $65(64)$ & $43(40)$ & $60.40 \pm 9.20(56.26 \pm 8.89)$ & $1049.4 \pm 159.9(977.4 \pm 154.5)$
\end{tabular}

${ }^{a}$ Values in parentheses are obtained through the PD1 method for assigning predissociative trajectories; see the text.

TABLE 4: Results for the Ar $+\mathrm{CH}_{4}$ Reaction Using the "ZPE-V Analysis" and the PD2 Method for Defining Predissociative Trajectories $^{a}$

\begin{tabular}{cllllll}
\hline$T / \mathrm{K}$ & \multicolumn{1}{c}{$N_{\text {tot }}$} & $N_{\mathrm{CH}_{4}^{*}}$ & $N_{600}$ & $N_{\text {diss }}$ & \multicolumn{1}{c}{$10^{4} \sigma_{\mathrm{CID}} / \AA^{2}$} & $k(T) / 10^{9} \mathrm{~cm}^{3} \mathrm{~s}^{-1} \mathrm{~mol}^{-1}$ \\
\hline 2500 & $5998755(5998724)$ & $105(88)$ & $33(31)$ & $2(1)$ & $0.17 \pm 0.12(0.08 \pm 0.08)$ & $0.2 \pm 0.2(0.1 \pm 0.1)$ \\
2800 & $998879(998850)$ & $93(78)$ & $38(37)$ & $3(1)$ & $1.51 \pm 0.87(0.50 \pm 0.50)$ & $2.1 \pm 1.2(0.7 \pm 0.7)$ \\
3000 & $498576(498544)$ & $108(80)$ & $42(39)$ & $3(3)$ & $3.02 \pm 1.75(3.02 \pm 1.75)$ & $4.3 \pm 2.5(4.3 \pm 2.5)$ \\
3200 & $496955(496903)$ & $213(183)$ & $63(59)$ & $8(8)$ & $8.09 \pm 2.86(8.09 \pm 2.86)$ & $11.9 \pm 4.2(11.9 \pm 4.2)$ \\
3500 & $246167(246099)$ & $242(194)$ & $92(88)$ & $7(7)$ & $14.29 \pm 5.40(14.30 \pm 5.40)$ & $21.9 \pm 8.3(21.9 \pm 8.3)$ \\
3800 & $96850(96803)$ & $187(156)$ & $69(65)$ & $6(6)$ & $31.14 \pm 12.71(31.16 \pm 12.72)$ & $49.7 \pm 20.3(49.7 \pm 20.3)$ \\
4000 & $38081(38061)$ & $103(91)$ & $39(38)$ & $5(5)$ & $66.00 \pm 29.51(66.03 \pm 29.53)$ & $108.1 \pm 48.4(108.2 \pm 48.4)$ \\
4200 & $37277(37245)$ & $137(114)$ & $52(52)$ & $11(10)$ & $148.33 \pm 44.72(134.96 \pm 42.67)$ & $249.0 \pm 75.1(226.5 \pm 71.6)$ \\
4500 & $35786(35740)$ & $202(172)$ & $65(64)$ & $12(11)$ & $168.55 \pm 48.65(154.71 \pm 46.64)$ & $292.9 \pm 84.5(268.8 \pm 81.0)$
\end{tabular}

${ }^{a}$ Values in parentheses are obtained through the PD1 method of assigning predissociative trajectories; see the text.

In the second approach (hereafter referred to as "ZPE analysis"), all trajectories ending with an internal energy below the ZPE for $\mathrm{CH}_{3}$ (i.e., $E_{\text {int }}<128.89 \mathrm{kcal} \mathrm{mol}^{-1}$ ) are considered to be nondissociative. However, because during the dissociation $\mathrm{CH}_{4}^{*} \rightarrow \mathrm{CH}_{3}+\mathrm{H}$ part of the $\mathrm{CH}_{4}^{*}$ internal energy may be channeled into translation, it is not possible, a priori, to know the number of energized species that will reach a $\mathrm{CH}_{3}$ product with its ZPE. Thus, all trajectories forming $\mathrm{CH}_{4}^{*}$ species, as defined by the "ZPE analysis", are continued until one of the $\mathrm{C}-\mathrm{H}$ bonds breaks down (i.e., $R_{\mathrm{C}-\mathrm{H}}>10 \AA$ ) or the integration time reaches $t=600 \mathrm{ps}$ (i.e., the average collision time estimated for the experimental conditions of Kiefer and Kumaran ${ }^{7}$ ); the results for the "ZPE analysis" are collected in Table 3. In this table, $N_{\mathrm{CH}_{4}^{*}}, N_{600}$, and $N_{\text {diss }}$ stand for the number of $\mathrm{CH}_{4}^{*}$ complexes formed in the $\mathrm{Ar}+\mathrm{CH}_{4}$ collision, the number of "nondissociating" trajectories, and those leading to $\mathrm{CH}_{3}$ with an internal energy above its ZPE, respectively. Note that the difference between $N_{\mathrm{CH}_{4}^{*}}$ and $N_{600}+N_{\text {diss }}$ corresponds to the number of trajectories dissociating to $\mathrm{CH}_{3}$ without internal energy above the respective ZPE, which are assigned as nonreactive in the "ZPE analysis". In turn, "nondissociating" trajectories correspond to extremely stable $\mathrm{CH}_{4}^{*}$ species that will last more than the estimated average collision time and, hence, are also considered to be nondissociative. We show in Figure 4 the logarithm of the decay rates for $\mathrm{CH}_{4}^{*}$ complexes that follow the PD2 criterion for assigning predissociative trajectories. It is apparent in this figure that the decay rates for $T=2500,3500$, and $4500 \mathrm{~K}$ present essentially an exponential behavior, although the energy distribution of $\mathrm{CH}_{4}^{*}$ complexes is far from forming a microcanonical ensemble. Additionally, we have continued the integration after the aforementioned deadline for "nondissociating" trajectories (indicated by the dashed line in Figure 4) up to $t=9000 \mathrm{ps}$ and noticed that some $\mathrm{CH}_{4}^{*}$ complexes dissociate after $t=600 \mathrm{ps}$, but only one forms $\mathrm{CH}_{3}$ with an internal energy above its $\mathrm{ZPE}$ for $T=3500$ $\mathrm{K}$ (the same happens for $T=3200$ and $3800 \mathrm{~K}$, although it is not shown in the figure); for the remaining temperatures, there is no trajectory dissociating with the $\mathrm{ZPE}$ of $\mathrm{CH}_{3}$ after $t=600$ ps. Note also that some $\mathrm{CH}_{4}^{*}$ complexes do not dissociate even within the long time period of $9000 \mathrm{ps}$, and they possibly correspond to trajectories with quasi-periodic motion that hardly decay to form products.

Finally, it can be argued that one should look at the vibrational energy rather than the internal energy for comparison with the ZPE of the $\mathrm{CH}_{3}$ product formed in the dissociation of the energized $\mathrm{CH}_{4}^{*}$ species. Accordingly, we have considered a third approach that is similar to the "ZPE analysis" but where the vibrational energy of $\mathrm{CH}_{3}$ substitutes its internal energy in the comparison with the corresponding ZPE; this is hereafter referred to as "ZPE-V analysis", and the main results from this 

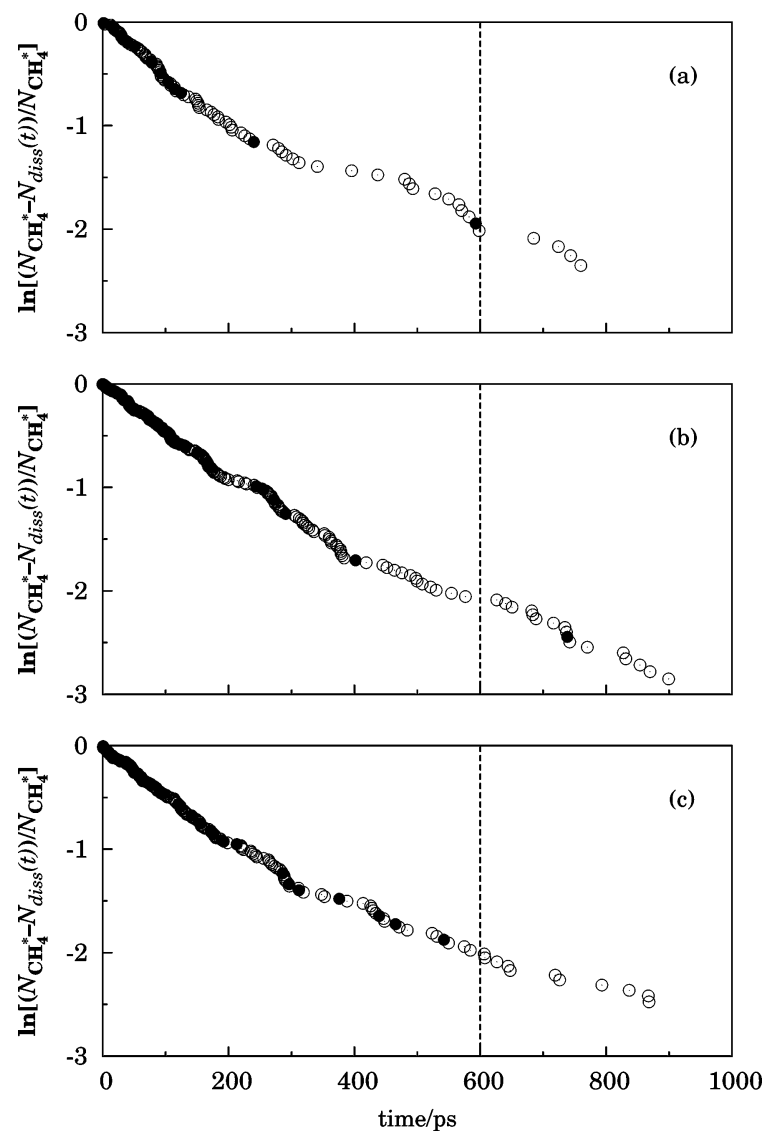

Figure 4. Logarithm of the decay rates as a function of time for the $\mathrm{CH}_{4}^{*}$ species that fulfill the PD2 criterion for the assignment of predissociative trajectories: (a) $T=2500 \mathrm{~K}$; (b) $T=3500 \mathrm{~K}$; (c) $T=$ $4500 \mathrm{~K}$. Full (open) circles correspond to trajectories that dissociate to form $\mathrm{CH}_{3}$ with an internal energy above (below) its $\mathrm{ZPE}$. The vertical dashed line sets $t=600 \mathrm{ps}$, which is used to establish the criterion for "nondissociating" trajectories in the present work. Though not shown by the time scale of the figure, trajectories are allowed to dissociate within $9000 \mathrm{ps}$; after that time, there are 10, 14, and 17 nondissociative trajectories for $T=2500,3500$, and $4500 \mathrm{~K}$, respectively. See the text for further explanation.

approach are summarized in Table 4. However, because of vibration-rotation coupling, the corresponding energies are not exactly separable, and it is possible to convert part of one into the other. Note that the final rotational energy $\left(\left\langle E_{\mathrm{r}}\right\rangle\right)$ is calculated in an approximate way by averaging the vibrational motion of the product molecule, and the corresponding vibrational counterpart results from the difference $E_{\text {int }}-\left\langle E_{\mathrm{r}}\right\rangle^{53}$

The total number of trajectories used in the statistical analysis, which is related to the way of assigning predissociative trajectories (see above), is shown in Tables 2-4 under the symbol $N_{\text {tot. }}$. Note that, for each temperature, $N_{\text {tot }}$ is smaller when using the PD1 approach for defining predissociative trajectories (values in parentheses) than when using the more accurate PD2 approach considered in this work. The discrepancy between both sets of values is more significant in the case of the pure classical analysis (see Table 2), which may be attributed to its smaller energy threshold in comparison with $128.89 \mathrm{kcal} \mathrm{mol}^{-1}$ for the "ZPE analysis" and the "ZPE-V analysis". Of course, a similar trend arises for the number of $\mathrm{CH}_{4}^{*}$ complexes formed in the $\mathrm{Ar}+\mathrm{CH}_{4}$ collision $\left(N_{\mathrm{CH}_{4}^{*}}\right)$. Note also that columns corresponding to $N_{\text {tot }}, N_{\mathrm{CH}_{4}^{*}}$, and $N_{600}$ are equal in Tables 3 and 4 because the only difference between the "ZPE analysis" and the "ZPE-V analysis" arises from the type of energy (internal or vibrational) used for comparison with the $\mathrm{ZPE}$ of $\mathrm{CH}_{3}$ after the dissociation of energized $\mathrm{CH}_{4}^{*}$ species.

Moreover, we show for completeness, in Tables 2-4, the corresponding values of the CID cross sections $\left(\sigma_{\mathrm{CID}}\right)$ and rate constants $[k(T)]$; the latter will be discussed in section 3.2. It is apparent from these tables that, for each temperature, $\sigma_{\mathrm{CID}}$ decreases as the ZPE criterion for trajectory analysis becomes more restrictive. In turn, the values of $\sigma_{\mathrm{CID}}$ tend to increase when using the PD2 assignment of predissociative trajectories despite the PD1 approach (values in parentheses), especially in the cases of classical analysis (Table 2) and "ZPE analysis" (Table 3); for the "ZPE-V analysis" (Table 3), where the number of dissociative trajectories is always very small, $\sigma_{\mathrm{CID}}$ is approximately constant or slightly increases (for $T=2500$, 2800, 4200, and $4500 \mathrm{~K}$ ) when passing from PD1 to PD2.

3.2. Thermal Rate Coefficients. One of the main goals of the present work is the study of ZPE effects in the thermal rate constants for the reaction $\mathrm{Ar}+\mathrm{CH}_{4} \rightarrow \mathrm{CH}_{3}+\mathrm{H}+$ Ar. For this, we calculated the thermal rate constant as a function of temperature by using the pure classical analysis and two methods for assigning predissociative trajectories, that is, PD1 and PD2 (see section 3.1); the calculated values are shown in column 5 of Table 2. Considering that the experimental values for the rate constant vary from $\sim 2 \times 10^{9} \mathrm{~cm}^{3} \mathrm{~mol}^{-1} \mathrm{~s}^{-1}$ at $T=2500$ $\mathrm{K}$ to $\sim 1 \times 10^{12} \mathrm{~cm}^{3} \mathrm{~mol}^{-1} \mathrm{~s}^{-1}$ at $T=4500 \mathrm{~K}$, we observe, from Table 2 , that the calculated values using the classical analysis of trajectories lead to discrepancies of about 2 orders of magnitude in the case where the PD1 approach has been considered (values given in parentheses); discrepancies of about 3 orders of magnitude are obtained when applying, in contrast, the PD2 method. Although the potential energy surface used here for the $\mathrm{ArCH}_{4}$ species may have some inaccuracies, one can hardly explain such a discrepancy based only on the potential model. In fact, the analytical $\mathrm{DHS}^{46}$ potential energy surface is expected to describe well the $\mathrm{C}-\mathrm{H}$ bond rupture, including the angular deformations of both methane and the methyl radical along the minimum energy path. Moreover, as described in section 2.1, we have improved the $\mathrm{DHS}^{46}$ potential energy surface to eliminate some of its unphysical behavior in describing the $\mathrm{H}-\mathrm{H}$ repulsion at small internuclear distances; also, the $\mathrm{Ar}-\mathrm{C}$ and $\mathrm{Ar}-\mathrm{H}$ pair potentials ${ }^{42}$ are expected to be accurate for describing the $\mathrm{Ar}-\mathrm{CH}_{4}$ interaction in the range of temperatures considered here. Thus, we believe that ZPE effects have a major influence on the thermal rate constants of the title reaction.

We investigate the importance of $\mathrm{ZPE}$ effects in the $\mathrm{CH}_{3}$ product by considering the passive approximations described as the "ZPE analysis" and the "ZPE-V analysis" in section 3.1. We recall here that the "ZPE analysis" considers nonreactive all of the dissociative trajectories forming $\mathrm{CH}_{3}$ products with an internal energy less than its ZPE, while the "ZPE-V analysis" uses, instead, the vibrational energy of $\mathrm{CH}_{3}$ for the same purpose. Note that, for the temperature range considered here, the $\mathrm{CH}_{4}$ reactant is always prepared with an energy content quite above the corresponding ZPE and, hence, is not expected to fall below that value after a nonreactive collision. Because of this, we think that it is useless to apply any ZPE constraint to $\mathrm{CH}_{4}$, as has been done in other works ${ }^{19,56,57}$ where the vibrational energy of the reactants is kept constant rather than being randomly selected from appropriate distributions.

The thermal rate constants obtained for reaction $\mathrm{Ar}+\mathrm{CH}_{4}$ $\rightarrow \mathrm{CH}_{3}+\mathrm{H}+\mathrm{Ar}$ by applying the "ZPE analysis" and excluding the predissociative trajectories identified with the PD2 method are represented in Figure 5. We give in Table 3 the correspond- 


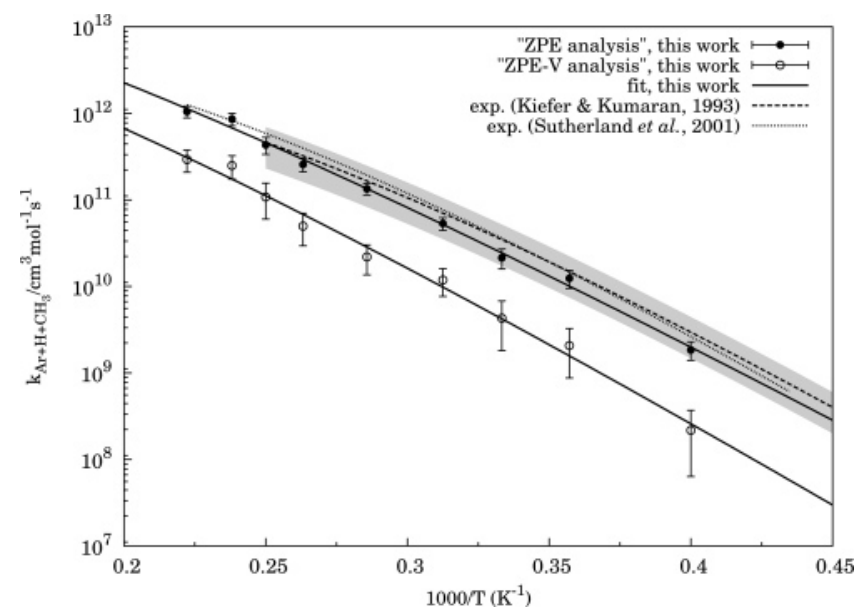

Figure 5. Thermal rate coefficient for the reaction $\mathrm{Ar}+\mathrm{CH}_{4} \rightarrow \mathrm{CH}_{3}$ $+\mathrm{H}+\mathrm{Ar}$ as a function of temperature. The points refer to the trajectory calculations: "ZPE analysis" (full dots) and "ZPE-V analysis" (open dots). The solid, thick lines correspond to least-squares fits of the calculated points using eq 14; error bars indicate the $68 \%$ confidence interval of the Monte Carlo integration. The experimental results are represented by the dashed (ref 11) and dotted (ref 12) lines; the 50\% error expected for the experimental results of Kiefer and Kumaran ${ }^{7}$ is shown by the shaded area. See the text for further explanation.

ing numerical values as well as those obtained by the PD1 approach (values given in parentheses). It is clear from this table that, in contrast with the pure classical analysis, both PD1 and PD2 approaches give almost the same values for the rate constants. Also shown in Figure 5 are the experimental curves of Kiefer and Kumaran ${ }^{7}$ and Sutherland et al. ${ }^{12}$ that have been fitted to the generalized Arrhenius expression

$$
k(T)=A T^{m} \exp (-E / R T)
$$

where $A, m$, and $E$ are fitting parameters. Although the experimental results from Sutherland et al. ${ }^{12}$ are for the $\mathrm{Kr}+$ $\mathrm{CH}_{4}$ reaction, they expect a little difference in the energy transfer for both systems (see also section 3.3) and, hence, the rate constants may be compared with each other. In fact, the values of the rate constants measured by Kiefer and Kumaran ${ }^{7}$ and Sutherland et al. ${ }^{12}$ agree within $\pm 25 \%$ for the temperature range $2300 \leq T / \mathrm{K} \leq 4500 . .^{12}$ In turn, the trajectory results arising from the "ZPE analysis" can also be fitted by using eq 14, and the parameters become $\log \left(A / \mathrm{cm}^{3} \mathrm{~mol}^{-1} \mathrm{~s}^{-1}\right)=28.7 \pm 13.4$, $m=-3.3 \pm 3.4$, and $E=92.8 \pm 22.4 \mathrm{kcal} \mathrm{mol}^{-1}$. Clearly, the agreement between the trajectory results from the "ZPE analysis" and the experiments is quite good, though slightly underestimating them, especially for lower temperatures. Note that even the experimental rate constants are not free from errors, being estimated as $\pm 50 \%$ (shaded area in the figure) for the determinations of Kiefer and Kumaran. ${ }^{7}$

Moreover, the rate constants calculated by the "ZPE-V analysis" and using the PD2 approach to identify predissociative trajectories are shown in Figure 5 (open dots), while the numerical values are given in Table 4. Also represented in this table are the corresponding values of $k(T)$ (given in parentheses) obtained by applying the PD1 approach. As in the "ZPE analysis", one can hardly observe significant differences in the values of $k(T)$ obtained by both the PD1 and PD2 approaches. We also show in Figure 5 the curve that fits the values calculated by the "ZPE-V analysis" to eq 14; the fitting parameters are $\log \left(A / \mathrm{cm}^{3} \mathrm{~mol}^{-1} \mathrm{~s}^{-1}\right)=29.4 \pm 23.1, m=-3.5 \pm 5.8$, and $E$ $=102.5 \pm 38.6 \mathrm{kcal} \mathrm{mol}^{-1}$. We observe in Figure 5 that the thermal rate constants from the "ZPE-V analysis" constitute a lower bound for both the experimental measurements and the values obtained with the "ZPE analysis". This is the expected result because the "ZPE-V analysis" is more restrictive than the "ZPE analysis". Finally, it must be pointed out that, though the "ZPE-V analysis" appears to be more physically acceptable (because one expects the vibrational energy rather than the internal energy to be above the $\mathrm{ZPE}$ of $\mathrm{CH}_{3}$ ), the "ZPE analysis" allows some flexibility in the energy criterion, which perhaps accounts for some lack of accuracy in the calculation of the vibrational energy (see section 3.1); thus, this may explain the good agreement between the values of $k(T)$ calculated from the "ZPE analysis" and those measured experimentally.

3.3. Collisional Energy Transfer. The $n$th moment of the energy transfer in all of the collisions can be obtained through trajectory calculations by using the expression

$$
\left\langle\Delta E^{n}\right\rangle_{\text {traj }}=\sum_{i=1}^{N_{\text {traj }} \Delta E_{i}^{n}} \frac{N_{\text {traj }}}{{ }_{\text {traj }}}
$$

where $N_{\text {traj }}$ is the total number of calculated trajectories for the energy-transfer analysis and $\Delta E_{i}$ is the difference between the initial internal energy of $\mathrm{CH}_{4}$ and its value at the end of trajectory $i$. Note that eq 15 can be used to calculate the average energy transfer in all collisions $\left(\langle\Delta E\rangle_{\text {traj }}\right)$ as well as the corresponding root-mean-squared energy $\left(\left\langle\Delta E^{2}\right\rangle_{\text {traj }}^{1 / 2}\right)$. A similar expression also applies for the average energy transfer in deactivating collisions (i.e., trajectories where the internal energy of $\mathrm{CH}_{4}$ diminishes), $\left\langle\Delta E_{\mathrm{d}}\right\rangle_{\text {traj. }}$. Moreover, it is well-established ${ }^{13,58-61}$ that, for a comparison with experimental data, the trajectory results obtained by eq 15 have to be scaled

$$
\left\langle\Delta E^{n}\right\rangle=\frac{\pi b_{\max }^{2}}{\pi \sigma_{\mathrm{LJ}}^{2} \Omega^{(2,2)^{*}}}\left\langle\Delta E^{n}\right\rangle_{\text {traj }}
$$

where $\sigma_{\mathrm{LJ}}$ is the Lennard-Jones (LJ) collision diameter and $\Omega^{(2,2)^{*}}$ is the LJ collision integral (which can be easily evaluated with the COLRATE code ${ }^{62}$ ). The standard error of the trajectory results has been estimated by a bootstrap analysis. ${ }^{63-65}$

We show in Figure 6 the values of energy-transfer parameters $\langle\Delta E\rangle$ (panel a), $\left\langle\Delta E_{\mathrm{d}}\right\rangle$ (panel b), and $\left\langle\Delta E^{2}\right\rangle^{1 / 2}$ (panel c), calculated by scaling the trajectory results (cf. eq 16) for each temperature (T). Also shown in Figure 6 are the recent results of Miller et al. ${ }^{11}$ using two different models for the master equation to deduce the energy-transfer parameters from the experimental data of Kiefer and Kumaran. ${ }^{7}$ One of these models (hereafter referred to as model I) considers the total energy of $\mathrm{CH}_{4}$ as an independent variable for four different forms of the energytransfer probability $P\left(E, E^{\prime}\right)$ (all shown by the full lines in the figure), while the other (model II) looks at its vibrational energy and uses an exponential form for $P\left(E, E^{\prime}\right)$ (dashed lines); see ref 11 for details. Additionally, the $\langle\Delta E\rangle$ values of Sutherland et al. ${ }^{12}$ deduced from their experiment on $\mathrm{Kr}+\mathrm{CH}_{4}$ (which they expect to be similar to those of $\mathrm{Ar}+\mathrm{CH}_{4}$ ) are also shown in Figure 6a.

From a first glance of Figure 6, we observe that our results are in reasonable agreement with the estimations of Miller et al. ${ }^{11}$ and, at least, in qualitative agreement with those of Sutherland et al. ${ }^{12}$ for $\mathrm{Kr}+\mathrm{CH}_{4}$. In particular, the trajectory results present a good agreement with model I for $\langle\Delta E\rangle$ and $\left\langle\Delta E^{2}\right\rangle^{1 / 2}$, while the calculated values of $\left\langle\Delta E_{\mathrm{d}}\right\rangle$ overestimate the results of Miller et al. ${ }^{11}$ In contrast, our results are in complete disagreement with the energy-transfer values obtained with model II, which has been considered to give too low values. ${ }^{11}$ 

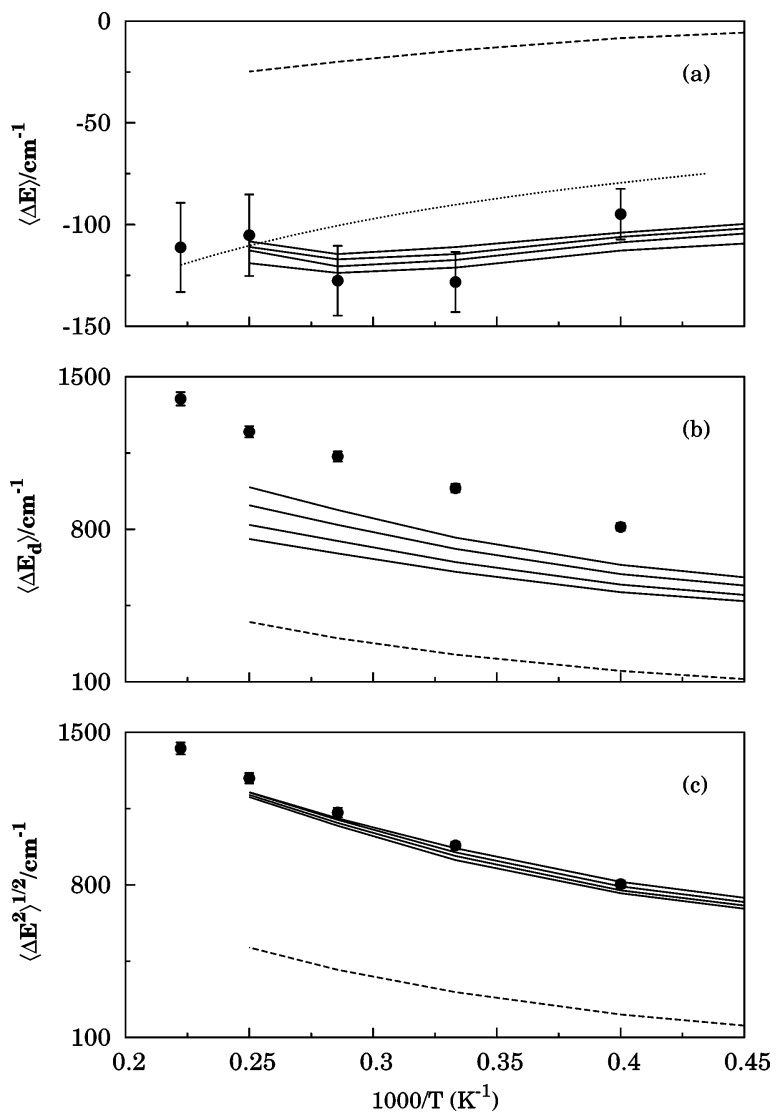

Figure 6. Energy-transfer parameters for $\mathrm{Ar}+\mathrm{CH}_{4}$ as a function of temperature: (a) $\langle\Delta E\rangle$; (b) $\left\langle\Delta E_{\mathrm{d}}\right\rangle$; (c) $\left\langle\Delta E^{2}\right\rangle^{1 / 2}$. The solid dots are the results of the present work obtained from eq 16; error bars are estimated by a bootstrap analysis. ${ }^{63-65}$ The results of Miller et al. ${ }^{11}$ are represented by the solid and dashed lines; the solid lines correspond to four different forms of the energy-transfer function $P\left(E, E^{\prime}\right)$, which depends on the total energy of $\mathrm{CH}_{4}$, while for the results represented in the dashed lines, $P\left(E, E^{\prime}\right)$ is an exponential-type function depending on the vibrational energy of $\mathrm{CH}_{4}$. Also shown by the dotted line (panel a) is the result of Sutherland et al. ${ }^{12}$ for $\mathrm{Kr}+\mathrm{CH}_{4}$. See the text for further explanation.

Another important feature from Figure 6 is that both $\left\langle\Delta E_{\mathrm{d}}\right\rangle$ and $\left\langle\Delta E^{2}\right\rangle^{1 / 2}$ vary more rapidly with temperature than $\langle\Delta E\rangle$. Thus, as has been advocated by Miller et al., ${ }^{11}$ assuming $\langle\Delta E\rangle$ (despite $\left.\left\langle\Delta E_{\mathrm{d}}\right\rangle\right)$ as independent of temperature may be a reasonable first approximation for modeling unimolecular reactions whenever better information is not available. Indeed, though presenting a slightly decreasing tendency (with some oscillations) as $T$ increases, our values of $\langle\Delta E\rangle$ are essentially constant within the error bars (Figure 6a), which is in agreement with the behavior shown by the results of Miller et al. ${ }^{11}$ (model I) and, moderately, with those of Sutherland et al. ${ }^{12}$ for $\mathrm{Kr}+$ $\mathrm{CH}_{4}$.

To gain more insight about the energy-transfer fluxes arising in the trajectory calculations, we have displayed in Figure 7 the average values of energy transfer in all of the collisions from rotation to vibration $\left(\mathrm{R} \rightarrow \mathrm{V}^{\prime}\right)$, rotation to translation ( $\mathrm{R}$ $\left.\rightarrow \mathrm{T}^{\prime}\right)$, and vibration to translation $\left(\mathrm{V} \rightarrow \mathrm{T}^{\prime}\right)$ as a function of temperature; these values have been calculated on the basis of simple balances among the three types of energy [i.e., vibration $(\mathrm{V})$, rotation $(\mathrm{R})$, and translation $(\mathrm{T})]$, taken on average for the six possible sets in which the trajectories may fall: (i) $\mathrm{V}$ activation; (ii) $\mathrm{V}, \mathrm{T}$ activation; (iii) $\mathrm{T}$ activation; (iv) $\mathrm{R}$ activation; (v) R, V activation; and (vi) R, T activation. It is clear from Figure 7 that the energy transfer among $\mathrm{CH}_{4}$ internal degrees of freedom $\left(\left\langle E_{R} \rightarrow v^{\prime}\right\rangle\right)$ is a strong function of temperature

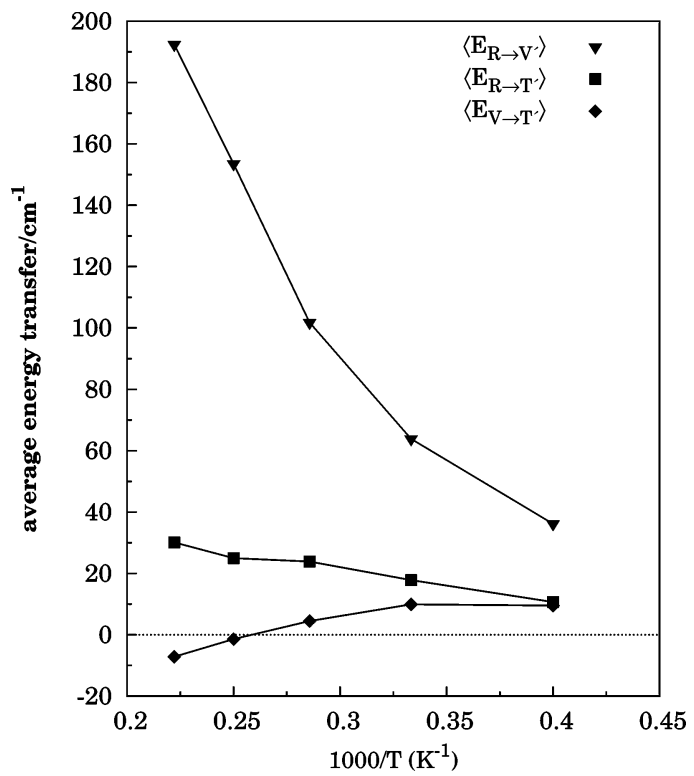

Figure 7. Average values of the energy transfer for rotation to vibration $\left(\left\langle E_{\mathrm{R} \rightarrow \mathrm{V}^{\prime}}\right\rangle\right)$, rotation to translation $\left(\left\langle E_{\mathrm{R} \rightarrow \mathrm{V}^{\prime}}\right\rangle\right)$, and vibration to translation $\left(\left\langle E_{\mathrm{V} \rightarrow \mathrm{T}^{\prime}}\right\rangle\right)$ as a function of temperature: triangles, $\left\langle E_{\mathrm{R} \rightarrow \mathrm{V}^{\prime}}\right\rangle$; squares, $\left\langle E_{\mathrm{R} \rightarrow \mathrm{T}^{\prime}}\right\rangle$; diamonds, $\left\langle E_{\mathrm{V} \rightarrow \mathrm{T}^{\prime}}\right\rangle$. The dotted line is depicted for reference; negative values of the energy transfer mean that the flux is in the reverse direction of the indicated one. See the text for further explanation.

and a significant amount of rotational energy is transferred to vibrational during the inelastic collision process, especially at high temperatures. This may be attributed to a very efficient $\mathrm{R}-\mathrm{V}$ coupling, which is normally improved as the initial rotational energy is raised, as is the case for increasing temperature (see section 2.2). Clearly, this indicates that rotational degrees of freedom must be treated as active, especially at high temperatures. Conversely, the variations of $\left\langle E_{\mathrm{R} \rightarrow \mathrm{T}^{\prime}}\right\rangle$ and $\left\langle E_{\mathrm{V} \rightarrow \mathrm{T}^{\prime}}\right\rangle$ with temperature are, in general, very small. Indeed, $\left\langle E_{\mathrm{R} \rightarrow \mathrm{T}^{\prime}}\right\rangle$ slightly increases with temperature, while $\left\langle E_{\mathrm{V} \rightarrow \mathrm{T}^{\prime}}\right\rangle$ appears to decrease at a larger rate (especially for high temperatures), which may be due to a stronger $\mathrm{V}-\mathrm{T}$ coupling as the collision energy increases (in an average sense) and the vibrational $\mathrm{CH}_{4}$ content decreases (see section 2.2); note also that the $\mathrm{V} \rightarrow \mathrm{T}^{\prime}$ flux is reversed (i.e., it becomes $\mathrm{T} \rightarrow \mathrm{V}^{\prime}$ ) at $T$ $\approx 4000 \mathrm{~K}$. Because $-\langle\Delta E\rangle$ depends on these two contributions, the reason becomes apparent for its nearly constant value with temperature, as described above (see also Figure 6a).

\section{Conclusions}

We have performed the first theoretical dynamics study of the $\mathrm{Ar}+\mathrm{CH}_{4}$ reaction where the rate constant has been calculated in the temperature range $2500 \leq T / \mathrm{K} \leq 4500$. The $\mathrm{CH}_{4}$ DHS surface ${ }^{46}$ has been improved by performing QCISD$(\mathrm{T}) / 6-311++\mathrm{G}(\mathrm{d}, \mathrm{p})$ ab initio calculations in order to account for $\mathrm{H}-\mathrm{H}$ repulsive interactions at high vibrational energies; in addition, the $\mathrm{Ar}-\mathrm{C}$ and $\mathrm{Ar}-\mathrm{H}$ pair potentials ${ }^{42}$ that have been added to model the $\mathrm{Ar}-\mathrm{CH}_{4}$ intermolecular interaction are accurate within the range of temperatures studied in this work. The results show that the $\mathrm{ZPE}$ of the $\mathrm{CH}_{3}$ product molecule has a major influence on the calculated thermal rate constants; after ZPE correction, the rate constants present a good agreement with the experimental ones, irrespective of the criterion (PD1 or PD2) used to assign predissociative trajectories. We have also estimated the energy-transfer parameters from trajectory calculations where the initial internal energy of $\mathrm{CH}_{4}$ has been fixed at the dissociation threshold and the collision energy selected from a thermal distribution. The values of $\langle\Delta E\rangle$ and 
$\left\langle\Delta E^{2}\right\rangle^{1 / 2}$ are in good agreement with those deduced by Miller et al. ${ }^{11}$ from the experiment, ${ }^{7}$ while overestimating them in the case of $\left\langle\Delta E_{\mathrm{d}}\right\rangle$. Finally, we have shown that the nearly constant value of $-\langle\Delta E\rangle$ over the studied range of temperatures results from a subtle balance due to an increase of the average $\mathrm{R} \rightarrow \mathrm{T}^{\prime}$ energy transfer and a slightly stronger decrease of the average $\mathrm{V} \rightarrow \mathrm{T}^{\prime}$ one.

Acknowledgment. The authors thank Dr. J. A. Miller for providing us with his numerical data for the energy transfers (Figure 6) and Dr. K. F. Lim for assistance on using the COLRATE code. ${ }^{62}$ The Portuguese-Spanish joint-action financial support from Conselho de Reitores das Universidades Portuguesas (Contract E-10/03) and Ministerio de Ciencia y Tecnología (Contract HP2002-0071) is gratefully acknowledged. E.M.-N. and A.F.-R. acknowledge Ministerio de Ciencia y Tecnología (Spain) for their Ramón y Cajal research contracts.

\section{References and Notes}

(1) Marcus, R. A. J. Chem. Phys. 1952, 20, 359.

(2) Marcus, R. A.; Rice, O. K. J. Phys. Colloid Chem. 1951, 55, 894.

(3) Warnatz, J. In Combustion Chemistry; Gardiner, W. C., Ed.; Springer-Verlag: New York, 1984; p 197.

(4) Roth, P.; Just, T. Ber. Bunsen-Ges. Phys. Chem 1975, 79, 682.

(5) Tabayashi, K.; Bauer, S. H. Combust. Flame 1979, 34, 63.

(6) Klemm, R. B.; Sutherland, J. W.; Rabinowitz, M. J.; Patterson, P.

M.; Quartemont, J. M.; Tao, W. J. Phys. Chem. 1992, 96, 1786.

(7) Kiefer, J. H.; Kumaran, S. S. J. Phys. Chem. 1993, 97, 414.

(8) Davidson, D. F.; Hanson, R. K.; Bowman, C. T. Int. J. Chem. Kinet.

1995, 27, 305

(9) Koike, T.; Kudo, M.; Maeda, I.; Yamada, H. Int. J. Chem. Kinet.

2000, 32, 1 .

(10) Cobos, C. J.; Troe, J. Z. Phys. Chem. (Muenchen) 1990, 167, 129.

(11) Miller, J. A.; Klippenstein, S. J.; Raffy, C. J. Phys. Chem. A 2002, $106,4904$.

(12) Sutherland, J. W.; Su, M.-C.; Michael, J. V. Int. J. Chem. Kinet. 2001, 33, 669 .

(13) Brown, N. J.; Miller, J. A. J. Chem. Phys. 1984, 80, 5568

(14) Lendvay, G.; Schatz, G. C. In Classical Trajectory Methods for Studying Energy Transfer from Highly Vibrationally Excited Molecules; Barker, R. J., Ed.; JAI Press: Greenwich, CT, 1995; Vol. 2B, p 481.

(15) Grinchak, M. B.; Levitsky, A. A.; Polak, L. S.; Umanskii, S. Y. Chem. Phys. 1984, 88, 365.

(16) Hase, W. L.; Date, N.; Bhuiyan, L. B.; Buckowski, D. G. J. Phys. Chem. 1985, 89, 2502.

(17) Hu, X.; Hase, W. L. J. Phys. Chem. 1988, 92, 4040.

(18) Varandas, A. J. C.; Brandão, J.; Pastrana, M. R. J. Chem. Phys. 1992, 96, 5137.

(19) Varandas, A. J. C.; Marques, J. M. C. J. Chem. Phys. 1992, 97, 4050 .

(20) Marques, J. M. C.; Varandas, A. J. C. An. Fis. 1994, 90, 284.

(21) Varandas, A. J. C. J. Chem. Phys. 1993, 99, 1076.

(22) Varandas, A. J. C. Chem. Phys. Lett. 1994, 225, 18

(23) Bowman, J. M.; Gadzy, B.; Sun, Q. J. Chem. Phys. 1989, 91, 2859.

(24) Miller, W. H.; Hase, W. L.; Darling, C. L. J. Chem. Phys. 1989 91, 2863. 1908 .

(25) Varandas, A. J. C.; Marques, J. M. C. J. Chem. Phys. 1994, 100,

(26) Ben-Nun, M.; Levine, R. D. J. Chem. Phys. 1994, 101, 8768

(27) Kumar, S.; Sathyamurthy, N.; Ramaswamy, R. J. Chem. Phys. 1995 103,6021

(28) Ben-Nun, M.; Levine, R. D. J. Chem. Phys. 1996, 105, 8136.

(29) Lim, K. F.; McCormack, D. A. J. Chem. Phys. 1995, 102, 1705.

(30) McCormack, D. A.; Lim, K. F. Phys. Chem. Chem. Phys. 1999, 1,

(31) Truhlar, D. G. J. Phys. Chem. 1979, 83, 18

(32) Shen, D.; Pritchard, H. O. J. Chem. Soc., Faraday Trans. 1996 92,4357

(33) Marks, A. J. J. Chem. Phys. 1998, 108, 1438

(34) Stock, G.; Müller, U. J. Chem. Phys. 1999, 111, 65.

(35) Müller, U.; Stock, G. J. Chem. Phys. 1999, 111, 77.

(36) Lim, K. F. J. Chem. Phys., Faraday Trans. 1997, 93, 669.

(37) Schlier, C. J. Chem. Phys. 1995, 103, 1989.

(38) McCormack, D. A.; Lim, K. F. J. Chem. Phys. 1995, 103, 1991.

(39) Shen, D.; Chan, W.-T.; Pritchard, H. O. J. Chem. Soc., Faraday Trans. 1995, 91, 3747

(40) Guo, Y.; Thompson, D. L.; Sewell, T. D. J. Chem. Phys. 1996 104, 576.

(41) Peslherbe, G. H.; Hase, W. L. J. Chem. Phys. 1996, 104, 9445.

(42) Sun, L.; Claire, P. S.; Meroueh, O.; Hase, W. L. J. Chem. Phys. 2001, 114, 535

(43) Rodrigues, S. P. J.; Varandas, A. J. C. J. Phys. Chem. A 1998, 102,6266

(44) Varandas, A. J. C.; Pais, A. A. C. C.; Marques, J. M. C.; Wang, W. Chem. Phys. Lett. 1996, 249, 264.

(45) Brunsvold, A. L.; Garton, D. J.; Minton, T. K.; Troya, D.; Schatz, G. C. J. Chem. Phys. 2004, 121, 11702 .

(46) Duchovic, R. J.; Hase, W. L.; Schlegel, H. B. J. Phys. Chem. 1984, $88,1339$.

(47) Liu, Y.; Jäger, W. J. Chem. Phys. 2004, 120, 9047.

(48) Heijmen, T. G. A.; Korona, T.; Moszynski, R.; Wormer, P. E. S.; van der Avoird, A. J. Chem. Phys. 1997, 107, 902.

(49) NIST Chemistry WebBook. http://webbook.nist.gov/chemistry/.

(50) Peslherbe, G. H.; Wang, H.; Hase, W. Adv. Chem. Phys. 1999, 105,171

(51) Marques, J. M. C.; Wang, W.; Pais, A. A. C. C.; Varandas, A. J. C. J. Phys. Chem. 1996, 100, 17513 .

(52) Marques, J. M. C.; Varandas, A. J. C. J. Phys. Chem. 1997, 101, 5168.

(53) Hase, W. L. MERCURY: A General Monte Carlo Classical Trajectory Computer Program; QCPE 453, 1983.

(54) Hase, W. L.; Buckowski, D. G. Chem. Phys. Lett. 1980, 74, 284

(55) Lim, K. F.; Gilbert, R. G. J. Phys. Chem. 1990, 94, 72

(56) Hu, X.; Hase, W. L. J. Phys. Chem. 1992, 96, 7535.

(57) Troya, D.; Pascual, R. Z.; Garton, D. J.; Minton, T. K.; Schatz, G. C. J. Phys. Chem. A 2003, 107, 7161 .

(58) Clarke, D. L.; Oref, I.; Gilbert, R. G.; Lim, K. F. J. Chem. Phys. 1992, 96, 5983

(59) Lim, K. F. J. Chem. Phys. 1994, 100, 7385.

(60) Lim, K. F. J. Chem. Phys. 1994, 101, 8756.

(61) Lenzer, T.; Luther, K. J. Chem. Phys. 1996, 105, 10944.

(62) Lim, K. F. QCPE Bull. 1994, 14, 3

(63) Efron, B. SIAM Rev. 1979, 21, 460

(64) Diaconis, P.; Efron, B. Sci. Am. 1983, 248, 96.

(65) Lim, K. F. PEERAN: $P\left(E, E^{\prime}\right)$ Error Analysis Program; Deakin University: Geelong, Australia, 1993. 